\title{
Pacific
}

Journal of

Mathematics

\section{SOME FINITE PROPERTIES FOR VERTEX OPERATOR} SUPERALGEBRAS

ChOngYing Dong AND JiAnZHI HAN 


\title{
SOME FINITE PROPERTIES FOR VERTEX OPERATOR SUPERALGEBRAS
}

\author{
CHONGYING DONG AND JIANZHI HAN
}

\begin{abstract}
Vertex operator superalgebras are studied and various results on rational vertex operator superalgebras are obtained. In particular, the vertex operator super subalgebras generated by the weight $\frac{1}{2}$ and weight 1 subspaces are determined. It is also established that if the even part $V_{\overline{0}}$ of a vertex operator superalgebra $V$ is rational, so is $V$.
\end{abstract}

\section{Introduction}

Vertex operator superalgebras, which are natural generalizations of vertex operator algebras, have been studied extensively in [Dong and Zhao 2005; 2006; Kac and Wang 1994; Li 1996b; 1996a; Xu 1998]. In this paper, we study certain finite properties of vertex operator superalgebras following [Dong et al. 1998b; Dong and Mason 2004; 2006; Mason 2011].

A vertex operator superalgebra $V=V_{\overline{0}} \oplus V_{\overline{1}}$ has even part $V_{\overline{0}}$ and odd part $V_{\overline{1}}$, where $V_{\overline{0}}$ consists of vectors of integral weights and $V_{\overline{1}}$ consists of vectors whose weights are half integers but not integers. So there is a canonical automorphism $\sigma$ of $V$ acting on $V_{\bar{i}}$ as $(-1)^{i}$ and $V_{\overline{0}}$ is a vertex operator algebra which is also a fixed point subalgebra of $V$. So a better understanding of the relationship between representation theories of $V$ and $V_{\overline{0}}$ is definitely useful for the study of orbifold theory; see [Dijkgraaf et al. 1989; Dong et al. 2000]. Even the orbifold theory for vertex operator algebras with order 2 automorphism has not been understood fully.

Rationality, which is an analogue of semisimplicity of associative and Lie algebras, is probably the most important concept in the representation theory of vertex operator superalgebra. We first establish that if $V_{\overline{0}}$ is rational, $V$ is rational, although we believe that the rationalities of $V$ and $V_{\overline{0}}$ are equivalent from the orbifold theory. The main tool consists of the associative algebras $A_{g, n}(V)$ for $n \in \frac{1}{2} \mathbb{Z}_{+}$, which are generalizations of $A_{g, n}(V)$ as introduced and studied in [Dong et al. 1998b] (also see [Zhu 1996; Kac and Wang 1994; Dong et al. 1998a; Dong et al.

Chongying Dong was supported by NSF grants and a faculty research fund from the University of California at Santa Cruz.

MSC2010: 17B65, 17B69.

Keywords: vertex operator superalgebra. 
1998c]), where $g$ is an automorphism of $V$ of finite order. It is established that $V$ is $g$-rational if and only if $A_{g, n}$ is a finite dimensional semisimple associative algebra for large $n$. This is the key result to prove the rationality of $V$ from the rationality of $V_{\overline{0}}$. Another characterization of rationality is given through the Ext functor.

Our investigation next centers around the vertex operator super subalgebras of $V$ generated by homogeneous subspaces of small weights. The vertex operator subalgebra generated by $V_{\frac{1}{2}}$ is a holomorphic vertex operator superalgebra $U$ associated to an infinite dimensional Clifford algebra built from a finite dimensional vector space with a nondegenerate symmetric bilinear form. This enables us to decompose $V$ as a tensor product $U \otimes U^{c}$ where $U^{c}$, whose weight $\frac{1}{2}$ subspace is zero, is the commutant of $U$ in $V$ [Frenkel and Zhu 1992; Lepowsky and Li 2004]. Moreover, the module categories of $V$ and $U^{c}$ are equivalent. To study $V_{1}$, we first need to understand the algebraic structure of $V_{1}$. Under the assumption that $V$ is rational or $\sigma$-rational together with $C_{2}$-cofiniteness, we are able to show that $V_{1}$ is a reductive Lie algebra, using the modular invariance results from [Dong and Zhao 2005; Zhu 1996], and the fact that $E_{2}(\tau)$ is not modular. Also, the rank of $V_{1}$ and the dimension of $V_{\frac{1}{2}}$ are controlled by the effective central charge. Furthermore, for any simple Lie subalgebra $\mathfrak{g}$ of $V_{1}$, the vertex operator subalgebra generated by $\mathfrak{g}$ is isomorphic to the vertex operator algebra $L(k, 0)$, which is the integrable highest weight module for the affine Kac-Moody algebra $\hat{\mathfrak{g}}$. We also give a rational vertex operator subalgebra, which is a tensor product of affine vertex operator algebras and a lattice vertex operator algebra, and whose weight one subspace is exactly $V_{1}$.

We should point out that most of the results in this paper have been obtained in the case where $V$ is a vertex operator algebra in [Dong et al. 1998b; Dong and Mason 2004; 2006; Mason 2011]. So the results of this paper can be regarded as a "super" analogues of results presented in [Dong et al. 1998b; Dong and Mason 2004; 2006; Mason 2011]. The main ideas and the broad outlines also follow from these papers. A lot of arguments are omitted if they are the same as in the case of vertex operator algebras. On the other hand, there is a new phenomenon in the super case. Namely, either rationality together with $C_{2}$-cofiniteness or $\sigma$-rationality together with $C_{2}$-cofiniteness implies that $V_{1}$ is reductive. This gives strong evidence that rationality, $\sigma$-rationality of $V$, and rationality of $V_{\overline{0}}$ are equivalent. But we have no idea how to establish this.

This paper is organized as follows. In Section 2, we recall various notions of twisted modules for a vertex operator superalgebra and $g$-rationality for any automorphism of finite order from [Frenkel et al. 1988; Zhu 1996; Dong et al. 1998a; Dong and Zhao 2006]. In Section 3, we define a series of associative algebras $A_{g, n}(V)$ for a vertex operator superalgebra $V$ and $n \in \mathbb{Z}_{+}$. We exhibit how to use $A_{n}(V)$ to prove rationality of $V$ from the rationality of $V_{\overline{0}}$. It is also shown that if $V$ is $C_{2}$-cofinite or rational, $V$ is finitely generated and the automorphism 
group $\operatorname{Aut}(V)$ is an algebraic group. Section 4 is devoted to the study of vertex operator super subalgebras generated by $V_{\frac{1}{2}}$. In Section 5 we show that if $V$ is rational or $\sigma$-rational together with $C_{2}$-cofiniteness then the weight one subspace $V_{1}$ is a reductive Lie algebra whose rank is bounded above by the effective central charge $\tilde{c}$. Consequently, $\operatorname{dim} V_{\frac{1}{2}}$ is bounded above by $2 \tilde{c}+1$. Section 6 deals with the vertex operator subalgebra of $V$ generated by $V_{1}$.

We make the assumption that the reader is familiar with the theory of vertex operator algebras as presented in [Borcherds 1986; Dong and Lepowsky 1993; Frenkel et al. 1988; Lepowsky and Li 2004].

\section{Basics}

In this section we give the definition of a vertex operator superalgebra and several notions of modules; cf. [Dong et al. 1997; Dong and Zhao 2006; Feingold et al. 1991; Frenkel et al. 1988; Li 1996b; Zhu 1996].

We first recall that a super vector space is a $\mathbb{Z}_{2}$-graded vector space $V=V_{\overline{0}} \oplus V_{\overline{1}}$. The elements in $V_{\overline{0}}$ (respectively $V_{\overline{1}}$ ) are called even (respectively odd). Let $\tilde{v}$ be 0 if $v \in V_{\overline{0}}$, and 1 if $v \in V_{\overline{1}}$.

Definition 2.1. A vertex operator superalgebra (VOSA) is a $\frac{1}{2} \mathbb{Z}$-graded vector space

$$
V=\bigoplus_{n \in \frac{1}{2} \mathbb{Z}} V_{n}=V_{\overline{0}} \oplus V_{\overline{1}}
$$

with $V_{\overline{0}}=\sum_{n \in \mathbb{Z}} V_{n}$ and $V_{\overline{1}}=\sum_{n \in \mathbb{Z}} V_{n+\frac{1}{2}}$ satisfying all the axioms in the definition of vertex operator algebra except that the Jacobi identity is replaced by

$$
\begin{aligned}
z_{0}^{-1} \delta\left(\frac{z_{1}-z_{2}}{z_{0}}\right) Y\left(u, z_{1}\right) Y\left(v, z_{2}\right)-(-1)^{\tilde{u} \tilde{v}} z_{0}^{-1} \delta & \left(\frac{-z_{2}+z_{1}}{z_{0}}\right) Y\left(v, z_{2}\right) Y\left(u, z_{1}\right) \\
& =z_{2}^{-1} \delta\left(\frac{z_{1}-z_{0}}{z_{2}}\right) Y\left(Y\left(u, z_{0}\right) v, z_{2}\right) .
\end{aligned}
$$

Throughout the paper we always assume that $V$ is a vertex operator superalgebra unless otherwise stated.

Definition 2.2. An automorphism $g$ of a VOSA $V$ is a linear automorphism of $V$ preserving the vacuum vector $\mathbf{1}$ and the conformal vector $\omega$ such that the actions of $g$ and $Y(v, z)$ on $V$ are compatible in the sense that

$$
g Y(v, z) g^{-1}=Y(g v, z)
$$

for $v \in V$.

Denote by $\operatorname{Aut}(V)$ the set consisting of all automorphisms of $V$. Observe that any automorphism of $V$ commutes with $L(0)$ and hence preserves each homogeneous subspace $V_{n}$. As a consequence, any automorphism preserves both $V_{\overline{0}}$ and 
$V_{\overline{1}}$. There is a canonical automorphism $\sigma$ of $V$ with $\sigma \mid V_{\bar{i}}=(-1)^{i}$ associated to the $\mathbb{Z}_{2}$-grading of $V$.

Let $g \in \operatorname{Aut}(V)$ with finite order $T$. Then we can decompose $V$ into eigenspaces of $g$ :

$$
V=\bigoplus_{r=0}^{T-1} V^{r},
$$

where $V^{r}=\left\{v \in V \mid g v=e^{-2 \pi i r / T} v\right\}$.

Definition 2.3. A weak $g$-twisted $V$-module $M$ is a $\mathbb{Z}_{2}$-graded vector space equipped with a linear map

$$
\begin{gathered}
Y_{M}: V \rightarrow(\text { End } M) \llbracket\left[z, z^{-1} \rrbracket,\right. \\
v \mapsto Y_{M}(v, z)=\sum_{n \in(1 / T) \mathbb{Z}} v_{n} z^{-n-1},
\end{gathered}
$$

such that, for all $u \in V^{r}(0 \leq r \leq T-1), v \in V$, and $w \in W$, the following conditions hold:

$$
\begin{aligned}
& Y_{M}(u, z)=\sum_{n \in r / T+\mathbb{Z}} u_{n} z^{-n-1}, \quad u_{n} w=0 \text { for } n \gg 0, \\
& Y_{M}(1, z)=\operatorname{Id}_{M},
\end{aligned}
$$

and

$$
\begin{array}{r}
z_{0}^{-1} \delta\left(\frac{z_{1}-z_{2}}{z_{0}}\right) Y_{M}\left(u, z_{1}\right) Y_{M}\left(v, z_{2}\right)-(-1)^{\tilde{u} \tilde{v}} z_{0}^{-1} \delta\left(\frac{-z_{2}+z_{1}}{z_{0}}\right) Y_{M}\left(v, z_{2}\right) Y_{M}\left(u, z_{1}\right) \\
=z_{2}^{-1} \delta\left(\frac{z_{1}-z_{0}}{z_{2}}\right)^{-r / T} \delta\left(\frac{z_{1}-z_{0}}{z_{2}}\right) Y_{M}\left(Y\left(u, z_{0}\right) v, z_{2}\right) .
\end{array}
$$

Definition 2.4. An admissible $g$-twisted $V$-module is a weak $g$-twisted $V$-module $M$ which carries a $(1 / T) \mathbb{Z}_{+}$-grading

satisfying

$$
M=\bigoplus_{n \in(1 / T) \mathbb{Z}_{+}} M(n)
$$

$$
v_{m} M(n) \subseteq M(n+\text { wt } v-m-1)
$$

for homogeneous $v \in V$ and $m \in \frac{1}{T} \mathbb{Z}$.

Definition 2.5. An ordinary $g$-twisted $V$-module is a weak $g$-twisted $V$-module

$$
M=\bigoplus_{\lambda \in \mathbb{C}} M_{\lambda}
$$

such that $\operatorname{dim} M_{\lambda}$ is finite and for fixed $\lambda, M_{n / T+\lambda}=0$ for all small enough integers $n$, where $M_{\lambda}=\{w \in M \mid L(0) w=\lambda w\}$ and $Y_{M}(\omega, z)=\sum_{n \in \mathbb{Z}} L(n) z^{-n-2}$. 
We say $V$ is $g$-rational if every admissible $g$-twisted $V$-module is completely reducible, that is, a direct sum of simple admissible $g$-twisted $V$-modules. $V$ is $g$-regular if the category of weak $g$-twisted $V$-modules is semisimple, namely, every weak $g$-twisted $V$-module is a direct sum of irreducible weak $g$-twisted $V$ modules. If $g=1$, we have the definitions of rationality and regularity for vertex operator superalgebras.

The following definitions are given for vertex operator algebras in [Dong and Mason 2006; Zhu 1996] and we extend these to vertex operator superalgebras here.

A vertex operator superalgebra $V$ is said to be of CFT type if the $L(0)$-grading on $V$ has no negative weights and the degree-zero homogeneous subspace $V_{0}$ is one-dimensional: in symbols, $V=\bigoplus_{n \in \frac{1}{2} \mathbb{Z}_{+}} V_{n}$ and $V_{0}=\mathbb{C} \mathbf{1}$. We say $V$ is of strong CFT type if $V$ satisfies the further condition $L(1) V_{1}=0 . V$ is said to be $C_{2}$-cofinite in the case where $C_{2}(V)$ has finite codimension in $V$, where $C_{2}(V)$ is the subspace of $V$ linearly spanned by all elements of the form $u_{-2} v$ for $u, v \in V$.

For convenience, let us introduce the term strongly g-rational for a simple vertex operator superalgebra $V$ which satisfies the following conditions:

(1) $V$ is of strong CFT type.

(2) $V$ is $C_{2}$-cofinite.

(3) $V$ is $g$-rational.

Definition 2.6. A bilinear form $(\cdot, \cdot)$ on a $V$-module $M$ is said to be invariant [Frenkel et al. 1993] if it satisfies the condition

$$
(Y(a, z) u, v)=\left(u, Y\left(e^{z L(1)}\left(e^{\pi i} z^{-2}\right)^{L(0)} a, z^{-1}\right) v\right) \quad \text { for } a \in V \text { and } u, v \in M .
$$

It is proved in [ $\mathrm{Li} \mathrm{1994;} \mathrm{Xu} 1998]$ that there exists a linear isomorphism from the space of invariant bilinear forms on $V$ to $\operatorname{Hom}_{\mathbb{C}}\left(V_{0} / L(1) V_{1}, \mathbb{C}\right)$. This implies that there is a unique, up to multiplication by a nonzero scalar, nondegenerate symmetric invariant bilinear form on $V$ if $V$ is simple and of strong CFT type.

\section{Rationality}

In this section we give a characterization of the rationality of a vertex operator superalgebra $V$ in terms of the rationality of a vertex operator subalgebra $V_{\overline{0}}$. We will show that if $V_{\overline{0}}$ is rational, $V$ is rational. We certainly believe that the converse is also true, that is, if $V$ is rational, $V_{\overline{0}}$ is also rational. This is similar to a wellknown conjecture in orbifold theory: Let $V$ be a rational vertex operator algebra, and $g$ is an order 2 automorphism of $V$. Then the fixed point vertex operator subalgebra is also rational. We will establish some other results on rationality. We also discuss the generators of $V$. 
The tool we use to prove the main result is the associative algebras $A_{n}(V)$, which is defined in [Dong et al. 1998c] for vertex operator algebra. Let $V$ be a vertex operator superalgebra. Let $O_{n}(V)$ be the subspace of $V$ linearly spanned by all $L(-1) u+L(0) u$ and $u \circ_{n} v$ where, for homogeneous $u \in V$ and $v \in V$,

$$
u \circ_{n} v= \begin{cases}\operatorname{Res}_{z} \frac{(1+z)^{\mathrm{wt} u+n}}{z^{2 n+2}} Y(u, z) v, & \text { if } u \in V_{\overline{0}}, \\ \operatorname{Res}_{z} \frac{(1+z)^{\mathrm{wt} u+n-\frac{1}{2}}}{z^{2 n+1}} Y(u, z) v, & \text { if } u \in V_{\overline{1}} .\end{cases}
$$

Define another operation $*_{n}$ on $V$ by

$$
u *_{n} v= \begin{cases}\sum_{m=0}^{n}(-1)^{m}\left(\begin{array}{c}
m+n \\
n
\end{array}\right) \operatorname{Res}_{z} \frac{(1+z)^{\mathrm{wt} u+n}}{z^{n+m+1}} Y(u, z) v, & \text { if } u, v \in V_{\overline{0}}, \\
0, & \text { if } u \in V_{\overline{1}} \text { or } v \in V_{\overline{1}} .\end{cases}
$$

Set $A_{n}(V)=V / O_{n}(V)$. Then $A_{0}(V)$ is the $A(V)$ studied in [Kac and Wang 1994]. Let $M$ be a weak $V$-module. Define the " $n$-th lowest weight vector" subspace of $M$ as

$$
\Omega_{n}(M)=\left\{w \in M \mid u_{\mathrm{wt} u+n+i} w=0, u \in V, i \geq 0\right\} .
$$

As in [Dong et al. 1998c] we have the following results.

Theorem 3.1. (1) Suppose that $M$ is a weak $V$-module. Then $\Omega_{n}(M)$ is an $A_{n}(V)$-module such that $a$ acts as $o(a)$ for $a \in V_{\overline{0}}$, where $o(a)$ is defined to be $a_{\mathrm{wt} a-1}$ for homogeneous $a \in V_{\overline{0}}$ and extends it linearly.

(2) Suppose $M=\bigoplus_{i \in \frac{1}{2} \mathbb{Z}_{+}} M(i)$ is an admissible $V$-module. Then

(a) $\Omega_{n}(M) \supset \bigoplus_{i \leq n} M(i)$;

(b) assuming $M$ is simple, $\Omega_{n}(M)=\bigoplus_{i \leq n} M(i)$, and each $M(i)$ is a simple $A_{n}(V)$-module for $i=0, \frac{1}{2}, \ldots, n$.

(3) $M \mapsto M(0)$ gives a bijection between irreducible admissible $V$-modules and simple $A(V)$-modules.

(4) The identity map induces an epimorphism from $A_{n}(V)$ to $A_{m}(V)$ for any $n \geq m$.

(5) If $V$ is g-rational, there are only finitely many irreducible admissible g-twisted $V$-modules up to isomorphism, and each irreducible admissible g-twisted $V$ module is ordinary.

Note that part (3) of the theorem was obtained in [Kac and Wang 1994].

The next lemma will be used as a characterization of the rationality of $V$ in terms of semisimplicity of $A_{n}(V)$ for large enough $n$.

Lemma 3.2. Suppose that $A(V)$ is finite dimensional. Then any admissible $V$ module is a direct sum of generalized eigenspaces for $L(0)$. 
Proof. Let $M=\bigoplus_{i \in \frac{1}{2} \mathbb{Z}_{+}} M(i)$ be an admissible $V$-module with $M(0) \neq 0$. Let $W$ be a maximal subspace of $M$ which is a direct sum of generalized eigenspaces with respect to $L(0)$. Then it is not hard to see that $W$ is a submodule of $M$. Consider the $A(V)$-module $M(0)$. By our assumption on finite dimension of $A(V)$, we see that there exists a nonzero simple $A(V)$-submodule of $M(0)$, on which $L(0)$ acts as a scalar by Schur's lemma. This shows that $W \neq 0$. We shall show $W=M$. Suppose $M / W \neq 0$. Choose the minimal $n \in \frac{1}{2} \mathbb{Z}_{+}$such that $M(n) / W(n) \neq 0$, where $W(n)=W \cap M(n)$. Then, by similar argument as above, we see that $M(n) / W(n)$ contains a nonzero simple $A(V)$-submodule, say $\mathscr{W}(n) / W(n) \neq 0$, where $\mathscr{W}(n)$ is a subspace of $M(n)$. Since both $\mathscr{W}(n) / W(n)$ and $W(n)$ are a direct sum of generalized eigenspaces for $L(0)$, so is $\mathscr{W}(n)$. Thus $\mathscr{W}(n) \subset W$ and $\mathscr{W}(n)=W(n)$, a contradiction.

Assume that $A(V)$ is finite dimensional. Let

$$
f(x)=\left(x-\lambda_{1}\right)\left(x-\lambda_{2}\right) \cdots\left(x-\lambda_{r}\right) \in \mathbb{C}[x]
$$

be the monic polynomial of least degree such that $f([w])=0$ in $A(V)$. Then, on any given simple $A(V)$-module, $L(0)$ must act as a constant $\lambda_{i}$ for some $i$. Note from Theorem 3.1 that $V$ has exactly $r$ inequivalent irreducible admissible modules $M^{i}=\sum_{n \in \frac{1}{2} \mathbb{Z}_{+}} M_{\lambda_{i}+n}^{i}$ for $i=1, \cdots, r$. Then there exists $m_{i}>0$ such that $M_{\lambda_{i}+n}^{i} \neq 0$ for all $n \geq m_{i}$. Let $N$ be a positive integer greater than $\left|\lambda_{i}-\lambda_{j}\right|$, $\left|\lambda_{i}\right|+1$, and $m_{i}$ for $i, j=1, \ldots, r$.

Note that the rationality is defined from the representation theory. It is always believed that such a property, which is analogous to the semisimplicity of Lie and associative algebras, should have its own internal characterization. The following result can be regraded as an internal characterization of rationality.

Theorem 3.3. $V$ is rational if and only if $A_{n}(V)$ is finite dimensional and semisimple for some $n \geq N$.

Proof. The proof of [Dong et al. 1998c, Theorem 4.10] shows that if $V$ is rational, $A_{n}(V)$ is semisimple and finite dimensional for all $n$. Now we assume that $A_{n}(V)$ is semisimple for some $n \geq N$. By Theorem 3.1, $A_{m}(V)$ is semisimple for all $m \leq n$. Let $M=\bigoplus_{i \in \frac{1}{2} \mathbb{Z}_{+}} M(i)$ be an admissible $V$-module with $M(0) \neq 0$. By Lemma 3.2, we can write

$$
M=\sum_{\lambda \in\left\{\lambda_{1}, \ldots, \lambda_{r}\right\}} \bigoplus_{n \in \frac{1}{2} \mathbb{Z}_{+}} M_{\lambda+n},
$$

where $M_{\lambda+n}$ is the generalized eigenspace for $L(0)$ with eigenvalue $\lambda+n$. Note that, for each $\lambda \in\left\{\lambda_{1}, \ldots, \lambda_{r}\right\}$, the subspace $\bigoplus_{n \in \frac{1}{2} \mathbb{Z}_{+}} M_{\lambda+n}$ is an admissible submodule of $M$. Without loss of generality, we may assume that $M=\bigoplus_{n \in \frac{1}{2} \mathbb{Z}_{+}} M(n)$ for some $\lambda \in\left\{\lambda_{1}, \ldots, \lambda_{r}\right\}$, where $M(n)=M_{\lambda+n}$. 
We assert that the submodule $W$ generated by $\bigoplus_{n \leq N, n \in \frac{1}{2} \mathbb{Z}_{+}} M(n)$ is equal to the entire $M$. Otherwise, $0 \neq M / W=\bigoplus_{n>N, n \in \frac{1}{2} \mathbb{Z}_{+}} M(n) / W(n)$, where $W(n)=$ $W \cap M(n)$. Let $n_{0} \in \frac{1}{2} \mathbb{Z}_{+}$be minimal such that $M\left(n_{0}\right) / W\left(n_{0}\right) \neq 0$. Then $n_{0}>N$ and $M\left(n_{0}\right) / W\left(n_{0}\right)$ is an $A(V)$-module by Theorem 3.1. Since $A(V)$ is semisimple, there exists a nonzero simple $A(V)$-submodule of $M\left(n_{0}\right) / W\left(n_{0}\right)$ on which $L(0)$ acts as the constant $\lambda+n_{0} \in\left\{\lambda_{1}, \ldots, \lambda_{r}\right\}$, which implies $\left|\lambda-\lambda_{j}\right|=n_{0}$ for some $j$. But this is impossible by our choice on $N$. Thus we must have $W=M$.

We next show that if $X$ is a simple $A(V)$-submodule, $X$ generates an irreducible $V$-module $U$. Denote by $J=\bigoplus_{n \in \frac{1}{2} \mathbb{Z}_{+}} J(n)$ the maximal submodule of $U$ such that $J(0)=0$, where $J(n)=J \cap U(n)$. Then the quotient $W=U / J$ is irreducible and $W(0)=X$. Since $\bigoplus_{0 \leq n \leq N} U(n)$ is a semisimple $A_{N}(V)$-module we can regard each $W(n)$ as an $A_{N}(V)$-submodule of $U(n)$ for $n \leq N$. From the choice of $N$, we know that $W(N) \neq 0$. Then the admissible $V$-submodule of $U$ generated by $W(N)$ contains $W(0)=X$. Thus $W(N)=U(N)$, so $J(N)=0$. By our choice of $N$, again we see that $J$ must be trivial. This implies that $U=W$ is irreducible.

It follows that the admissible $V$-submodule $\mathcal{W}$ of $M$ generated by $M(0)$ is completely reducible. Note that $M(1)=\mathscr{W}(1) \oplus P$, where $P$ is a semisimple $A(V)$ module. Again the admissible submodule of $M$ generated by $P$ is completely reducible. Continuing in this way completes the proof.

Remark 3.4. Even in the case where $V$ is a vertex operator algebra, Theorem 3.3 strengthens [Dong et al. 1998c, Theorem 4.11], where we require that $A_{n}(V)$ is semisimple for all $n$.

Remark 3.5. There is a twisted analogue $A_{g, n}(V)$ (cf. [Dong et al. 1998b]) of $A_{n}(V)$. One can similarly define the positive integer $N_{g}$. Then Theorem 3.3 still holds, that is, $V$ is $g$-rational if and only if $A_{g, n}(V)$ is finite dimensional and semisimple for some $n \geq N_{g}$.

We now use Theorem 3.3 to prove the following result.

Proposition 3.6. Let $V=V_{\overline{0}} \oplus V_{\overline{1}}$ be a VOSA. If $V_{\overline{0}}$ is rational, $V$ is rational.

Proof. Suppose $V_{\overline{0}}$ is rational. Then, by Theorem 3.3, $A_{n}\left(V_{\overline{0}}\right)$ is a finite dimensional semisimple associative algebra if $n$ is sufficiently large. This implies that $A_{n}(V)$ is semisimple as $A_{n}(V)$ is a quotient of $A_{n}\left(V_{\overline{0}}\right)$. Applying Theorem 3.3 again yields that $V$ is rational.

We remark that we do not know how to prove the rationality of $V$ from the rationality of $V_{\overline{0}}$ without using $A_{n}(V)$. It is certainly a very interesting problem to find a different approach that does not use $A_{n}(V)$. Although we can not show the converse of Proposition 3.6, we strongly believe that rationalities of $V$ and $V_{\overline{0}}$ are equivalent. 
In the rest of this section we use the extension functor to consider the rationality of a vertex operator superalgebra $V$. This approach has been studied in [Abe 2005] for vertex operator algebra, but our rationality result is different from that given in [ibid.].

First let us describe the set $\operatorname{Ext}_{V}^{1}\left(M^{2}, M^{1}\right)$ for any weak $V$-module $M^{1}$ and $M^{2}$. We call a weak $V$-module $M$ an extension of $M^{2}$ by $M^{1}$ if there is a short exact sequence $0 \rightarrow M^{1} \rightarrow M \rightarrow M^{2} \rightarrow 0$. Two extensions $M$ and $N$ of $M^{2}$ by $M^{1}$ are said to be equivalent if there exists a $V$-homomorphism $f: M \rightarrow N$ such that the following diagram commutes:

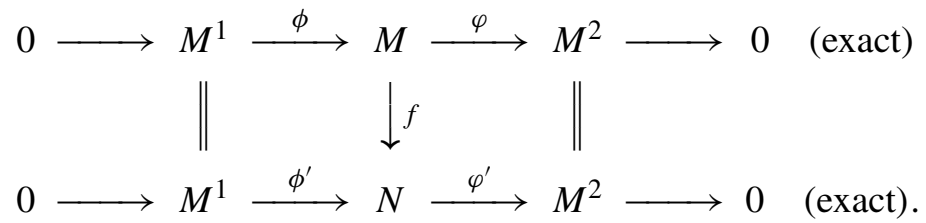

Define $\operatorname{Ext}_{V}^{1}\left(M^{2}, M^{1}\right)$ to be the set of all equivalent classes of $M^{2}$ by $M^{1}$. It is well known that $\operatorname{Ext}_{V}^{1}\left(M^{2}, M^{1}\right)$ carries the structure of an abelian group such that the equivalent class of $M^{1} \oplus M^{2}$ is the zero element.

Here is another equivalent condition of rationality.

Proposition 3.7. Let $V$ be a vertex operator superalgebra. Then $V$ is rational if and only if the following two conditions hold.

(a) Every admissible $V$-module contains a nontrivial irreducible admissible submodule.

(b) For any irreducible $V$-modules $M$ and $N$, $\operatorname{Ext}_{V}^{1}(M, N)=0$.

Proof. It is clear that rationality implies both (a) and (b). Now we assume that (a) and (b) hold. Let $M=\bigoplus_{n \in \frac{1}{2} \mathbb{Z}_{+}} M(n)$ be a nonzero admissible $V$-module. Let $W$ be the sum of irreducible admission $V$-submodules of $M$. Then $W=\bigoplus_{i \in I} W^{i}$, where each $W^{i}$ is an irreducible admissible $V$-module. By condition (a), $W \neq 0$. We assert that $W=M$. Otherwise consider the quotient module $M / W$. It follows from condition (a) again that there exists a weak $V$-submodule $M^{\prime}$ such that $M^{\prime} \supsetneq W$ and $M^{\prime} / W$ is an irreducible admissible $V$-module. Then by condition (b) and the properties of Ext, we have

$$
\operatorname{Ext}_{V}^{1}\left(M^{\prime} / W, W\right)=\bigoplus_{i \in I} \operatorname{Ext}_{V}\left(M^{\prime} / W, W^{i}\right)=0,
$$

that is, $M^{\prime}=M^{\prime} / W \oplus W$ as $V$-modules, contradicting the maximality of $W$. So the assertion is true and $M$ is a direct sum of irreducible admissible $V$-modules.

We now turn our attention to the generators of vertex operator superalgebras. 
Proposition 3.8. Let $V$ be a vertex operator superalgebra.

(a) If $V$ is rational or $C_{2}$-cofinite, $V$ is finitely generated.

(b) If $V$ is finitely generated, $\operatorname{Aut}(V)$ is an algebraic group.

These results were obtained in the case of vertex operator algebras in [Dong and Zhang 2008; Karel and Li 1999]; see also [Gaberdiel and Neitzke 2003; Dong and Griess 2002]. The same proof works here.

\section{Vertex operator subalgebra generated by $V_{\frac{1}{2}}$}

In this section we study the vertex operator super subalgebra $U$ of $V$ generated by $V_{\frac{1}{2}}$, and decompose $V$ as a tensor product $U \otimes U^{c}$, where $U$ is holomorphic in the sense that $U$ is the only irreducible module for itself and $U^{c}$, whose weight $\frac{1}{2}$ subspace is 0 , is the commutant of $U$ in $V$. This decomposition reduces the study of vertex operator superalgebras to the study of vertex operator superalgebras whose weight $\frac{1}{2}$ subspaces are 0 .

Let $V$ be a simple vertex operator superalgebra of strong CFT type. Then there is a unique invariant, symmetric, and nondegenerate bilinear form $(\cdot, \cdot)$ such that

$$
(\mathbf{1}, \mathbf{1})=\sqrt{-1}
$$

see [Li 1994; Xu 1998]. Then, for $u, v \in V_{\frac{1}{2}}$, one has

$$
u_{0} v=(u, v) \mathbf{1}
$$

and

$$
[u(m), v(n)]_{+}=(u, v) \delta_{m+n+1,0} .
$$

Note that the restriction of $(\cdot, \cdot)$ to $V_{\frac{1}{2}}$ is still nondegenerate. Let $\left\{a^{1}, a^{2}, \ldots, a^{l}\right\}$ be an orthonormal basis of $V_{\frac{1}{2}}$ with respect to the form $(\cdot, \cdot)$, where $l=\operatorname{dim} V_{\frac{1}{2}}$.

Let $U$ be the vertex super subalgebra of $V$ generated by $V_{\frac{1}{2}}$. Then, using (4-3), we see that

$$
U=\operatorname{Span}\left\{u_{-n_{1}}^{1} u_{-n_{2}}^{2} \cdots u_{-n_{r}}^{r} \mathbf{1} \mid u^{i} \in V_{\frac{1}{2}}, n_{1} \geq n_{2} \geq \cdots \geq n_{r}>0 \text { and } r \in \mathbb{Z}_{+}\right\} .
$$

In fact, $U$ carries the structure of a vertex operator superalgebra with conformal vector

$$
\omega^{\prime}=\frac{1}{2} \sum_{i=1}^{l} a_{-2}^{i} a_{-1}^{i} \mathbf{1} .
$$

Define operators $L^{\prime}(n)$ for $n \in \mathbb{Z}$ by

$$
Y\left(\omega^{\prime}, z\right)=\sum_{n \in \mathbb{Z}} w_{n}^{\prime} z^{-n-1}=\sum_{n \in \mathbb{Z}} L^{\prime}(n) z^{-n-2} .
$$


Then the weight $n$ subspace $U_{n}$ for $L^{\prime}(0)$ is given by

$$
\begin{array}{r}
U_{n}=\left\langle u_{-n_{1}}^{1} u_{-n_{2}}^{2} \cdots u_{-n_{r}}^{r} \mathbf{1}\right| u^{i} \in V_{\frac{1}{2}}, n_{1} \geq n_{2} \geq \cdots \geq n_{r}>0, r \in \mathbb{Z}_{+}, \\
\left.\quad \text { and } n_{1}+n_{2} \cdots+n_{r}=n+\frac{r}{2}\right\rangle .
\end{array}
$$

It is well known (cf. [Kac and Wang 1994]) that the vertex operator algebra $U$ generated by $V_{\frac{1}{2}}$ is holomorphic. So for any admissible $V$-module $M$, we can decompose $M$ into irreducible $U$-modules as follows

$$
M=U \otimes \bar{M}
$$

where $\bar{M}=\left\{w \in M \mid u_{n} w=0\right.$ for all $u \in U$ and $\left.n \in \mathbb{Z}_{+}\right\}$is the multiplicity space of $U$ in $M$. If $M=V$, the multiplicity space $\bar{M}$ is denoted by $U^{c}$ and is called the commutant of $U$ in $V$. In particular, $V=U \otimes U^{c}$. The $U^{c}$ is a vertex operator superalgebra (see [Frenkel and Zhu 1992; Lepowsky and Li 2004]) with $\omega-\omega^{\prime}$ as its conformal vector and $U_{\frac{1}{2}}^{c}=0$.

Let $\operatorname{Irr}(V)$ and $\operatorname{Irr}\left(U^{c}\right)$ denote the sets of the isomorphism classes of admissible irreducible $V$-modules and $U^{c}$-modules, respectively. The following result is straightforward.

Proposition 4.1. Let $V$ be a vertex operator superalgebra.

(a) For any admissible $V$-module $M . \bar{M}$ is an admissible $U^{c}$-module. Moreover, $M$ is irreducible if and only if $\bar{M}$ is irreducible.

(b) The map $U \otimes *: \operatorname{Irr}\left(U^{c}\right) \rightarrow \operatorname{Irr}(V)$ is a bijection.

(c) $V$ is rational if and only if $U^{c}$ is rational.

\section{The structure of weight 1 subspace}

In this section we will investigate the Lie algebra structure of weight 1 subspace $V_{1}$ and show that $V_{1}$ is a reductive Lie algebra if $V$ is $\sigma$-rational, using the modular invariance results obtained in [Dong and Zhao 2006]. We also find an upper bound for the rank of $V_{1}$ in terms of effective central charge. Similar results for vertex operator algebras were given previously in [Dong and Mason 2004], and the proof presented here is a modification of that used in [ibid.]. We also apply these results to estimate the dimension of weight $\frac{1}{2}$ subspace $V_{\frac{1}{2}}$ of $V$.

First, we need to discuss vertex operator superalgebras on the torus [Zhu 1996; Dong and Zhao 2005], vector-valued modular forms [Knopp and Mason 2003], and the modular invariance of trace functions [Zhu 1996; Dong and Zhao 2005].

Let $V$ be a vertex operator superalgebra. The vertex operator superalgebra

$$
(V, Y[v, z], \mathbf{1}, \tilde{\omega})
$$


on a torus (see [Zhu 1996; Dong and Zhao 2005]) is defined as follows:

$$
\begin{aligned}
Y[v, z] & =Y\left(v, e^{z}-1\right) e^{w t v}=\sum_{n \in \mathbb{Z}} v[n] z^{-n-1}, \\
Y[\tilde{w}, z] & =\sum_{n \in \mathbb{Z}} L[n] z^{-n-2}
\end{aligned}
$$

for homogeneous $v$ and $\tilde{\omega}=\omega-c / 24$.

We denote the eigenspace of $L[0]$ with eigenvalue $n \in \frac{1}{2} \mathbb{Z}$ by $V_{[n]}$. If $v \in V_{[n]}$, we write $\mathrm{wt}[v]=n$.

A holomorphic vector-valued modular form of weight $k$ (where $k$ is any real number) on the modular group $\Gamma=\operatorname{SL}(2, \mathbb{Z})$ may be described as follows: for any integer $p \geq 1$ it is a tuple $\left(T_{1}(\tau), \ldots, T_{p}(\tau)\right)$ of functions holomorphic in the complex upper half-plane together with a $p$-dimensional complex representation $\rho: \Gamma \rightarrow \operatorname{GL}(p, \mathbb{C})$ satisfying the following conditions.

(a) For all $\gamma \in \Gamma$ we have

$$
\left.\left(T_{1}, \ldots, T_{p}\right)^{t}\right|_{k} \gamma(\tau)=\rho(\gamma)\left(T_{1}(\tau), \ldots, T_{p}(\tau)\right)^{t}
$$

(where $t$ refers to the transpose of vectors and matrices).

(b) Each function $T_{j}(\tau)$ has a convergent $q$-expansion holomorphic at infinity:

$$
T_{j}(\tau)=\sum_{n \geq 0} a_{n}(j) q^{n / N_{j}}
$$

for positive integer $N_{j}$. (Here and below, $q=\exp 2 \pi i \tau$ ).

The following result plays an important role in this section.

Proposition 5.1 [Knopp and Mason 2003]. Let $\left(T_{1}, \ldots, T_{p}\right)$ be a holomorphic vector-valued modular form of weight $k$ associated to a representation $\rho$ of $\Gamma$. Then there is a nonnegative constant $\alpha$ depending only on $\rho$ such that the Fourier coefficients $a_{n}(j)$ satisfy the polynomial growth condition $a_{n}(j)=O\left(n^{k+2 \alpha}\right)$ for every $1 \leq j \leq p$.

Fix automorphisms $g, h$ of $V$ of finite orders. Let $M$ be a simple $g \sigma$-twisted $V$-module. Then

$$
M=\bigoplus_{n=0}^{\infty} M_{\lambda+n / T^{\prime}}
$$

for some $\lambda$ called the conformal weight of $M\left(M_{\lambda} \neq 0\right)$, where $T^{\prime}$ is the order of $g \sigma$. Suppose $M$ is $\sigma h$-stable, which is equivalent to the existence of a linearly isomorphic map $\phi(\sigma h): M \rightarrow M$ such that

$$
\phi(\sigma h) Y_{M}(v, z) \phi(\sigma h)^{-1}=Y_{M}((\sigma h) v, z)
$$


for all $v \in V$. From now on we assume that $V$ is $C_{2}$-cofinite. Then a function $F_{M}$ which is linear in $v \in V$ is defined for homogeneous $v \in V$ as follows:

$$
F_{M}(v, \tau)=q^{\lambda-c / 24} \sum_{n=0}^{\infty} \operatorname{tr}_{M_{\lambda+n / T}} o(v) \phi(\sigma h) q^{n / T}=\operatorname{tr}_{M} o(v) \phi(\sigma h) q^{L(0)-c / 24}
$$

which is a holomorphic function in the upper half-plane [Dong and Zhao 2005]. Here and below we write $F_{M}(\tau)$ rather than $F_{M}(\mathbf{1}, \tau)$ for simplicity. Then for any $u, v$ in $V$ such that $g v=h v=v$, we have

$$
\begin{aligned}
\operatorname{tr}_{M} o(u) o(v) \phi(\sigma h) q^{L(0)-c / 24} & \\
& =F_{M}(u[-1] v, \tau)-\sum_{k \geq 1} E_{2 k}(q) F_{M}(u[2 k-1] v, \tau) ;
\end{aligned}
$$

see [Dong and Zhao 2005; Zhu 1996]. The functions $E_{2 k}(\tau)$ are the Eisenstein series of weight $2 k$ :

$$
E_{2 k}(q)=\frac{-B_{2 k}}{2 k !}+\frac{2}{(2 k-1) !} \sum_{n=1}^{\infty} \sigma_{2 k-1}(n) q^{n},
$$

where $\sigma_{k}(n)$ is the sum of the $k$-powers of the divisors of $n$, and $B_{2 k}$ is a Bernoulli number. The $E_{2}(\tau)$ enjoys an exceptional transformation law. Namely, its transformation with respect to the matrix

$$
S=\left(\begin{array}{rr}
0 & -1 \\
1 & 0
\end{array}\right)
$$

has the form

$$
E_{2}\left(\frac{-1}{\tau}\right)=\tau^{2} E_{2}(\tau)-\frac{\tau}{2 \pi i} .
$$

We also need results on 1-point functions on the torus [Dong and Zhao 2005]. Let $g, h$ be automorphisms of $V$ of finite order. The space of $(g, h) 1$-point functions $\mathscr{C}(g, h)$ is the $\mathbb{C}$-linear space consisting of functions

$$
S: V \times \mathbb{H} \rightarrow \mathbb{C}
$$

(where $\mathbb{W}$ is the upper half-plane) satisfying certain conditions; see [Dong and Zhao 2005] for details. The following results can be found in [ibid.].

Theorem 5.2. Let $V$ be $C_{2}$-cofinite and $g, h \in \operatorname{Aut}(V)$ of finite orders.

(1) For $S \in \mathscr{C}(g, h)$ and

$$
\gamma=\left(\begin{array}{ll}
a & b \\
c & d
\end{array}\right) \in \Gamma
$$

we define

$$
\left.S\right|_{\gamma}(v, \tau)=\left.S\right|_{k}(v, \tau)=(c \tau+d)^{-k} S(v, \tau)
$$


for $v \in V_{[k]}$, and extend linearly. Then $\left.\left.S\right|_{\gamma} \in \mathscr{C}((g, h) \gamma)\right)$, where $(g, h) \gamma=$ $\left(g^{a} h^{c}, g^{b} h^{d}\right)$.

(2) Let $M$ be a simple $g \sigma$-twisted $V$-module such that $M$ is $h$ and $\sigma$-stable. Then $F_{M}(v, \tau) \in \mathscr{C}(g, h)$.

(3) Suppose that $V$ is go-rational and $M^{1}, \ldots, M^{m}$ are the inequivalent, simple $g \sigma$-twisted $V$-module such that $M^{i}$ is $h$ and $\sigma$-stable. Let $F_{1}, \ldots, F_{m}$ be the corresponding trace functions defined by (5-1). Then $F_{1}, \ldots, F_{m}$ form a basis of $\mathscr{C}(g, h)$.

We now assume that $V$ is of strong CFT type. Recall from [Frenkel et al. 1993] that the weight 1 subspace $V_{1}$ of $V$ carries a natural Lie algebra structure, the Lie bracket being given by $[u, v]=u_{0} v$ for $u, v \in V_{1}$. Then any weak $V$-module is automatically a $V_{1}$-module such that $v \in V_{1}$ acts as $v_{0}$. Note that there is a unique nondegenerate symmetric invariant bilinear form $\langle\cdot, \cdot\rangle$ such that $\langle\mathbf{1}, \mathbf{1}\rangle=-1$ and the restriction of $\langle\cdot, \cdot\rangle$ to $V_{1}$ endows $V_{1}$ with a nondegenerate, symmetric, invariant bilinear form such that $u_{1} v=\langle u, v\rangle \mathbf{1}$ for $u, v \in V_{1}$.

The following two theorems are extensions of similar results from vertex operator algebras [Dong and Mason 2004] to vertex operator superalgebras.

Theorem 5.3. Let $V$ be strongly rational or strongly $\sigma$-rational. Then the Lie algebra $V_{1}$ is reductive.

Proof. We first deal with the case where $V$ is $\sigma$-rational. We have to show that the nilpotent radical $N$ of the Lie algebra $V_{1}$ is zero. Suppose not, and take any nonzero element $u \in N$. Each $V_{i}$ for $i \in \frac{1}{2} \mathbb{Z}$ is a finite dimensional $V_{1}$-module and has a composition series $0=W^{0} \subset W^{1} \subset W^{2} \subset W^{3} \subset \cdots \subset$ such that $u_{0}$ acts trivially on each composite factor $W^{i} / W^{i-1}(i=1,2, \cdots)$. Note that we can take $\phi(\sigma)=\sigma$ on $V$. Thus $V$ is $\sigma$-stable. In fact, any irreducible $V$-module is $\sigma$-stable; see [Dong and Zhao 2005, Lemma 6.1]. As a result, $\operatorname{tr}_{V_{i}} o(u) o(v) \sigma=0$ for all $v \in V_{1}$ and $i \in \frac{1}{2} \mathbb{Z}$. It follows from (5-2) that

$$
F_{V}(u[-1] v, \tau)=\sum_{k \geq 1} E_{2 k}(\tau) F_{V}(u[2 k-1] v, \tau),
$$

where $(g, h)=(\sigma, 1)$ and $F_{V} \in \mathscr{C}(\sigma, 1)$, by Theorem 5.2.

Note that if $k>1$ is an integer, the element $u[2 k-1] v$ has $L[0]$-weight $2-2 k<0$ and hence is 0 . The nondegeneracy of the bilinear form $\langle\cdot, \cdot\rangle$ guarantees that there exists $v \in V_{1}$ such that $\langle u, v\rangle=1$. With this choice of $v$, (5-4) simplifies to read

$$
F_{V}(u[-1] v, \tau)=E_{2}(\tau) F_{V}(\tau) .
$$

By Theorem 3.1, $V$ has finitely many irreducible $\sigma$-twisted $V$-modules up to isomorphism. We denote these modules by $M^{1}, \ldots, M^{m}$. Note from Theorem 5.2 
that the

$$
S=\left(\begin{array}{cc}
0 & 1 \\
-1 & 0
\end{array}\right) \in \Gamma
$$

maps $\mathscr{C}(\sigma, 1)$ to $\mathscr{C}(1, \sigma)$. By Theorem 5.2 again we see that

$$
F_{V}\left(u[-1] v,-\frac{1}{\tau}\right)=\tau^{2} \sum_{i=1}^{m} s_{i} F_{M^{i}}(u[-1] v, \tau)
$$

and

$$
F_{V}\left(-\frac{1}{\tau}\right)=\sum_{i=1}^{m} s_{i} F_{M^{i}}(\tau)
$$

for some $s_{i} \in \mathbb{C}$. Similar to equality (5-5), we also have

$$
F_{M^{i}}(u[-1] v, \tau)=E_{2}(\tau) F_{M^{i}}(\tau) \text { for } i=1, \ldots, m .
$$

Thus

$$
\begin{aligned}
\tau^{2} \sum_{i=1}^{m} s_{i} F_{M^{i}}(u[-1] v, \tau) & =F_{V}\left(u[-1] v, \frac{-1}{\tau}\right) \\
& =E_{2}\left(\frac{-1}{\tau}\right) F_{V}\left(\frac{-1}{\tau}\right) \\
& =\left(\tau^{2} E_{2}(\tau)-\frac{\tau}{2 \pi i}\right) \sum_{i=1}^{m} s_{i} F_{M^{i}}(\tau) \\
& =\tau^{2} \sum_{i=1}^{m} s_{i} F_{M^{i}}(u[-1] v, \tau)-\frac{\tau}{2 \pi i} \sum_{i=1}^{m} s_{i} F_{M^{i}}(\tau) .
\end{aligned}
$$

Of course, the equality (5-3) is involved in the calculations above. Canceling the term $\tau^{2} \sum_{i=1}^{m} s_{i} F_{M^{i}}(u[-1] v, \tau)$ gives rise to the identity $\sum_{i=1}^{m} s_{i} F_{M^{i}}(\tau)=0$, which in turn implies $F_{V}(-1 / \tau)=0$. But this is clearly not true, since

$$
F_{V}\left(\frac{-1}{\tau}\right)=q^{-c / 24}\left(\sum_{n \in \mathbb{Z}}\left(\operatorname{dim} V_{n}\right) q^{n}-\sum_{n \in \frac{1}{2}+\mathbb{Z}}\left(\operatorname{dim} V_{n}\right) q^{n}\right) \neq 0 .
$$

So $N=0$ and $V_{1}$ is reductive.

Now we assume that $V$ is rational. As before we need to show that the nilpotent radical $N$ of the Lie algebra $V_{1}$ is zero. This time we use $\mathscr{C}(\sigma, \sigma)$ instead of $\mathscr{C}(\sigma, 1)$ and $\mathscr{C}(1, \sigma)$. In this case, the $S \in \Gamma$ maps $\mathscr{C}(\sigma, \sigma)$ to itself. A similar argument applies.

Remark 5.4. It is proved in [Dong and Mason 2004] that if a vertex operator algebra is strongly rational, weight one subspace is reductive. If one can prove the 
rationality of $V_{\overline{0}}$ from the rationality and $\sigma$-rationality of $V$, Theorem 5.3 follows immediately. Unfortunately, none of these results have been established.

The following result will be used in the next section.

Lemma 5.5. Let $V$ be a vertex operator superalgebra.

(a) If $V$ is strongly rational, any admissible $V$-module is a completely reducible $V_{1}$-module. This is also equivalent to saying the action of any Cartan subalgebra of the Lie algebra $V_{1}$ is semisimple on any admissible $V$-module.

(b) If $V$ is strongly $\sigma$-rational, any admissible $\sigma$-twisted $V$-module is a completely reducible $V_{1}$-module.

(c) If $V$ is either strongly rational or strongly $\sigma$-rational, any irreducible $\sigma^{i}$ twisted $V$-module is a completely reducible $V_{1}$-module for $i=0,1$.

Proof. Since the proof of (b) is similar to that of (a), we only show (a) and (c) for strongly rational vertex operator superalgebra $V$. Let $H$ be a Cartan subalgebra of $V_{1}$. It is enough to show that $H$ acts semisimply on any irreducible $\sigma^{i}$-twisted $V$ module for $i=0,1$. Since the homogeneous subspaces of an irreducible $\sigma^{i}$-twisted $V$-module are always finite dimensional, there is a common eigenvector of $H$ on the irreducible module. So it is enough to show that $H$ acts on $V$ semisimply.

First we show that for any nonzero $u \in H, h_{0}$ is not nilpotent. Note that the restriction of the bilinear form $\langle\cdot, \cdot\rangle$ to $H$ is nondegenerate. If $u_{0}$ is nilpotent for some nonzero $u \in H$, we can take $v \in H$ such that $\langle u, v\rangle=1$. The proof of Theorem 5.3 then gives a contradiction.

We now prove that $u_{0}$ is semisimple on $V$. Since $\operatorname{Aut}(V)$ is an algebraic group by Proposition 3.8, and $\left\{e^{t u_{0}} \mid t \in \mathbb{C}\right\}$ is a one-dimensional algebraic subgroup of $\operatorname{Aut}(V)$, we immediately see that $\left\{e^{t u_{0}} \mid t \in \mathbb{C}\right\}$ is isomorphic to the one-dimensional multiplicative algebraic group $\mathbb{C}_{m}$ as $u_{0}$ is not nilpotent; cf. [Mason 2011].

Now that $V_{1}$ is reductive, there are two extreme cases: $V_{1}$ is a semisimple Lie algebra, and $V_{1}$ is abelian. The vertex operator subalgebra generated by $V_{1}$ will be extensively investigated in Section 6. We study the rank of $V_{1}$ in the rest of this section. Let $l$ be the rank of $V_{1}$, that is, $l$ is the dimension of a Cartan subalgebra $H$ of $V_{1}$. Similar to the case of vertex operator algebras in [Dong and Mason 2004], $l$ is closely related to the effective central charge $\tilde{c}$, which is defined as follows: Let $\left\{M^{1}, \ldots, M^{m}\right\}$ be the irreducible $\sigma$-twisted $V$-modules up to isomorphism. Then there exist $\lambda_{i} \in \mathbb{C}$ such that $M^{i}=\sum_{n \in \frac{1}{2} \mathbb{Z}_{+}} M_{\lambda_{i}+n}^{i}$ with $M_{\lambda_{i}}^{i} \neq 0$. The $\lambda_{i}$ is called the conformal weight of $M^{i}$. By [Dong and Zhao 2005, Theorem 8.9], $\lambda_{i}$ and the central charge $c$ of $V$ are rational numbers for all $i$. Define $\lambda_{\min }$ to be the minimum of the conformal weights $\lambda_{i}$, and set

$$
\tilde{c}=c-24 \lambda_{\min }, \quad \tilde{\lambda}_{i}=\lambda_{i}-\lambda_{\min } .
$$

Theorem 5.6. Let $V$ be strongly $\sigma$-rational. Then $l \leq \tilde{c}$. 
Proof. Let $H$ be a Cartan subalgebra of $V_{1}$. Note that the component operators of the vertex operators $Y(u, z)$ on $V$ for $u \in H$ form a Heisenberg Lie algebra. This amounts to saying that for $u, v \in H$ the following relations hold:

$$
\left[u_{m}, v_{n}\right]=m \delta_{m,-n}\langle u, v\rangle \text {. }
$$

In fact, these relations also hold true on any $\sigma$-twisted $V$-module $M$.

Consider $(g, h)=(1,1)$. Let $F_{i}=F_{M^{i}}$ be as defined in (5-1). Then $F_{i} \in \mathscr{C}(1,1)$. Recall that

$$
\eta(\tau)=q^{1 / 24} \prod_{n=1}^{\infty}\left(1-q^{n}\right)
$$

is a modular form of weight $\frac{1}{2}$. Then

$$
\eta(\tau)^{\tilde{c}} F_{i}(\tau)=q^{\tilde{\lambda}_{i}} \prod_{n=1}^{\infty}\left(1-q^{n}\right)^{\tilde{c}} \sum_{n=0}^{\infty} \operatorname{tr}_{M_{\lambda_{i}+n / 2}} \phi(\sigma) q^{n / 2}
$$

is holomorphic in $\mathbb{U} \cup\{i \infty\}$. Now it follows from the transformation law for $\eta(\tau)$ and Theorem 5.2 that the $m$-tuple

$$
\left(\eta(\tau)^{\tilde{c}} F_{1}(\tau), \ldots, \eta(\tau)^{\tilde{c}} F_{m}(\tau)\right)
$$

is a holomorphic vector-valued modular form of weight $\tilde{c} / 2$. So the Fourier coefficients of $\eta(\tau)^{\tilde{c}} F_{i}(\tau)$ have polynomial growth by Proposition 5.1.

The Stone-von Neumann theorem provides us a somewhat different way to look more closely at $F_{i}(\tau)$. Namely, $M^{i}$ has the following tensor decomposition:

$$
M^{i}=M(1) \otimes_{\mathbb{C}} \Omega_{M^{i}},
$$

where $M(1)=\mathbb{C}\left[u_{m} \mid u \in H, m>0\right]$ is the Heisenberg vertex operator algebra of rank $l$ generated by $H$ and $\Omega_{M^{i}}=\left\{w \in M^{i} \mid u_{n} w=0\right.$ for $u \in H$ and $\left.n>0\right\}$. Then the trace function $F_{i}(\tau)$ corresponding to the decomposition (5-7) is equal to

$$
q^{(l-c) / 24} \eta(\tau)^{-l} \operatorname{tr}_{\Omega_{i}} \phi(\sigma) q^{L(0)},
$$

as $\operatorname{tr}_{M(1)} \phi(\sigma) q^{L(0)}=q^{l / 24} \eta(\tau)^{-l}$. Thus

$$
\eta(\tau)^{\tilde{c}} F_{i}(\tau)=q^{(l-c) / 24} \eta(\tau)^{\tilde{c}-l} \operatorname{tr}_{\Omega_{i}} \phi(\sigma) q^{L(0)} .
$$

We know the Fourier coefficients of the left side of (5-8) have polynomial growth. This forces the same to be true on $\eta(\tau)^{\tilde{c}-l}$. Then one has $\tilde{c}-l \geq 0$, as $\eta(\tau)^{s}$ has exponential growth of Fourier coefficients whenever $s<0$; cf. [Knopp 1970].

We now use Theorem 5.6 to do an estimation on the dimension of $V_{\frac{1}{2}}$.

Corollary 5.7. Let $V$ be strongly $\sigma$-rational. Then $\operatorname{dim} V_{\frac{1}{2}} \leq 2 \tilde{c}+1$. 
Proof. Let $d$ be a nonnegative integer such that $2 d \leq \operatorname{dim} V_{\frac{1}{2}} \leq 2 d+1$. Then there exists a unique (up to a constant) nondegenerate bilinear form satisfying (4-1). We point out that the restriction of $(\cdot, \cdot)$ to $V_{\frac{1}{2}}$ is still nondegenerate. So we can choose elements $b^{i}, b^{i^{*}} \in V_{\frac{1}{2}}$ such that $\left(b^{i}, b^{j^{*}}\right)^{2}=\delta_{i j}$ and $\left(b^{i}, b^{j}\right)=0=\left(b^{i^{*}}, b^{j^{*}}\right)$ for all $1 \leq i, j \leq d$. Set $h^{i} \stackrel{\frac{2}{=}}{=} b_{-1}^{i}\left(b^{i}\right)_{-1}^{*} \mathbf{1}$ for $i=1, \ldots, d$. Then $h^{i} \in V_{1}$ and $h_{1}^{i} h^{j}=\delta_{i, j}$. $h_{0}^{i} h^{j}=0$ for $i, j \in\{1, \cdots, d\}$. As a result, $\sum_{i=1}^{d} \mathbb{C} h^{i} \subset V_{1}$ is contained in a Cartan subalgebra of $V_{1}$. By Corollary 5.7, $d \leq l \leq \tilde{c}$, and the proof is complete.

\section{6. $C_{2}$-cofiniteness and integrability}

We continue our discussion on the weight 1 subspace $V_{1}$. We will determine the vertex operator subalgebra $\left\langle V_{1}\right\rangle$ of $V$ generated by $V_{1}$ following the approach in [Dong and Mason 2006]. It turns out that $\left\langle V_{1}\right\rangle$ is isomorphic to

$$
L_{\mathfrak{g}_{1}}\left(k_{1}, 0\right) \otimes \cdots \otimes L_{\mathfrak{g}_{s}}\left(k_{s}, 0\right) \otimes M(1),
$$

where $V_{1}=\mathfrak{g}_{1} \oplus \cdots \oplus \mathfrak{g}_{s} \oplus Z\left(V_{1}\right), \mathfrak{g}_{i}$ are simple, $k_{i} \geq 1$ are integers, and $M(1)$ is the Heisenberg vertex operator algebra built up from $Z\left(V_{1}\right)$ (see below for the definition of $\left.L_{\mathfrak{g}}(k, 0)\right)$. Moreover, $\left\langle V_{1}\right\rangle$ is contained in the rational vertex operator subalgebra $L_{\mathfrak{g}_{1}}\left(k_{1}, 0\right) \otimes \cdots \otimes L_{\mathfrak{g}_{s}}\left(k_{s}, 0\right) \otimes V_{L}$ for some positive definite lattice $L \subset Z\left(V_{1}\right)$ satisfying $\operatorname{rank}(L)=\operatorname{dim} Z\left(V_{1}\right)$.

Here we need to review the construction of untwisted affine Kac-Moody Lie algebras $\hat{\mathfrak{g}}$ associated with simple Lie algebras $\mathfrak{g}$ and relevant results from [Kac 1990]. Let $\mathfrak{h}$ be a Cartan subalgebra of $\mathfrak{g}$ and $\Phi$ the corresponding root system. Fix a nondegenerate symmetric invariant bilinear form $(\cdot, \cdot)$ on $\hat{\mathfrak{g}}$ such that the square length of a long root is 2 , where we have identified $\mathfrak{h}$ with its dual via the bilinear form. Then the affine Kac-Moody algebra associated to $\mathfrak{g}$ is given by

$$
\hat{\mathfrak{g}}=\mathfrak{g} \otimes \mathbb{C}\left[t, t^{-1}\right] \oplus \mathbb{C} K
$$

with the bracket relations

$$
[u(m), v(n)]=[u, v](m+n)+m(u, v) \delta_{m+n, 0} K \quad \text { and } \quad[K, \hat{\mathfrak{g}}]=0
$$

for $u, v \in \mathfrak{g}$ and $m, n \in \mathbb{Z}$, where $u(m)=u \otimes t^{m}$. Let $L(\lambda)$ be the irreducible $\mathfrak{g}$-module with highest weight $\lambda \in \mathfrak{h}$. Consider $L(\lambda)$ as a $\mathfrak{g} \otimes \mathbb{C}[t]$-module with $\mathfrak{g} \otimes \mathbb{C} t[t]$ acting trivially and with $K$ acting as the scalar $k \in \mathbb{C}$. Then the generalized Verma module

$$
V(k, \lambda)=\operatorname{Ind}_{\mathfrak{g}}^{\hat{\mathfrak{g}}} L(\lambda)=U(\hat{\mathfrak{g}}) \otimes_{U(\mathfrak{g} \otimes \mathbb{C}[t] \oplus \mathbb{C} K)} L(\lambda)
$$

has the unique irreducible quotient $L(k, \lambda)$. It is well known that $L(k, \lambda)$ is integrable if and only if $k$ is a nonnegative integer and $\lambda$ is a dominant integral weight such that $(\lambda, \theta) \leq k$, where $\theta \in \Phi$ is the maximal root. 
Let $V$ be a VOSA of strong CFT type and let $\langle\cdot, \cdot\rangle$ be the unique nondegenerate bilinear form satisfying $\langle\mathbf{1}, \mathbf{1}\rangle=-1$. Suppose that $\mathfrak{g} \subset V_{1}$ is a simple subalgebra. Then both bilinear forms $(\cdot, \cdot)$ and $\langle\cdot, \cdot\rangle$ on $\mathfrak{g}$ are symmetric and invariant, so they must be proportional, that is,

$$
\langle\cdot, \cdot\rangle=k(\cdot, \cdot) \quad \text { for some } k \in \mathbb{C} .
$$

Then, for any $u, v \in V_{1}$ and integers $m, n$, one has

$$
\left[u_{m}, v_{n}\right]=[u, v]_{m+n}+m u_{1} v \delta_{m+n, 0} .
$$

Comparing this with (6-1) shows that the map

$$
u(m) \rightarrow u_{m} \text { for } u \in \mathfrak{g} \text { and } m \in \mathbb{Z}
$$

together with $K \rightarrow k$ gives rise to a representation of $\hat{\mathfrak{g}}$ of level $k$.

Now we are going to state our main result related to $C_{2}$-integrability, which has already been proved to be true in [Dong and Mason 2006] for vertex operator algebras satisfying $C_{2}$-cofiniteness. But given a vertex operator superalgebra $V=$ $V_{\overline{0}} \oplus V_{\overline{1}}$ which satisfies the $C_{2}$-cofinite condition, generally, we can not prove that the even part $V_{\overline{0}}$ also has such a property. So in this sense, the following result sharpens [Dong and Mason 2006, Theorem 3.1], although the idea is similar.

Theorem 6.1. Let $V$ be a simple vertex operator superalgebra which is $C_{2}$-cofinite of strong CFT type, with $\mathfrak{g} \subset V_{1}$ a simple Lie subalgebra, $k$ the level of $V$ as $\hat{\mathfrak{g}}$ module, and the vertex operator subalgebra $U$ of $V$ generated by $\mathfrak{g}$. Then:

(a) The restriction of $\langle\cdot, \cdot\rangle$ to $\mathfrak{g}$ is nondegenerate.

(b) $U \cong L(k, 0)$.

(c) $k$ is a positive integer.

(d) $V$ is an integrable $\hat{\mathfrak{g}}$-module.

Proof. Let $\mathfrak{h}$ be a Cartan subalgebra of $\mathfrak{g}$, and $\mathfrak{g}=\mathfrak{h} \oplus \sum_{\alpha \in \Phi} \mathfrak{g}_{\alpha}$ the corresponding Cartan decomposition of $\mathfrak{g}$. Since $\mathfrak{g}$ is generated by subalgebras isomorphic to $\operatorname{sl}(2, \mathbb{C})$, it is good enough to show the theorem for $\mathfrak{g}=\operatorname{sl}(2, \mathbb{C})$. Let $\{h, x, y\}$ be the standard basis of $\mathfrak{g}$. Then $(\alpha, \alpha)=2$ and $k=\langle\alpha, \alpha\rangle / 2$ from this and Equation (6-2).

Clearly, $U=\langle\mathfrak{g}\rangle$ is a quotient of $V(k, 0)$. So $U$ is a $\hat{\mathfrak{g}}$-integrable module if and only if $U=L(k, 0)$ for some $k \in \mathbb{Z}_{+}$. This is also equivalent to the existence of a positive integer $r$ such that

$$
\left(x_{-1}\right)^{r} \mathbf{1}=0 .
$$

The proof of (6-3) is similar to the same result in [Dong and Mason 2006] and we omit the proof. (b) then immediately follows. Also note that $\mathfrak{g} \subset U$, so $U$ can not be a one-dimensional trivial module. Thus $k \neq 0$ and $k$ must be a positive 
integer, proving (c) and (a). Since $L(k, 0)$ is rational (cf. [Dong et al. 1997]), $V$ is a direct sum of irreducible $L(k, 0)$-modules, each of which is integrable as $\hat{\mathfrak{g}}$ module. Hence $V$ is an integrable $\hat{\mathfrak{g}}$-module. This proves (d).

Next we consider a toral subalgebra of $V_{1}$. Let $V$ be strongly rational or strongly $\sigma$-rational, and let $\mathfrak{h} \subset V_{1}$ be a toral subalgebra such that the restriction of $\langle\cdot, \cdot\rangle$ to $\mathfrak{h}$ remains nondegenerate. Notably, any Cartan subalgebra of $V_{1}$ automatically satisfies such a condition.

Theorem 6.2. Suppose $V$ is strongly rational or strongly $\sigma$-rational. Let $\mathfrak{h} \subset V_{1}$ be a toral subalgebra such that the restriction of $\langle\cdot, \cdot\rangle$ to $\mathfrak{h}$ is nondegenerate. Then there exists a positive-definite even lattice $L \subset \mathfrak{h}$ with rank $\operatorname{dim} \mathfrak{h}$ and a vertex operator super subalgebra $U$ of $V$ such that $\mathfrak{h} \subset U \cong V_{L}$.

This theorem has been proved in [Dong and Mason 2006] for vertex operator algebras; see also [Mason 2011]. The same argument using Lemma 5.5 is also valid for vertex operator superalgebras.

We now assume that

$$
V_{1}=\mathfrak{g}_{1} \oplus \cdots \oplus \mathfrak{g}_{s} \oplus \mathfrak{h},
$$

where $\mathfrak{g}_{i}$ are simple Lie algebras and $Z\left(V_{1}\right)=\mathfrak{h}$. By Theorems 5.3, 6.1, and 6.2 we have the following; see [Dong and Mason 2006; Mason 2011].

Corollary 6.3. The $V$ contains a strongly rational vertex operator subalgebra

$$
U=L_{\mathfrak{g}_{1}}\left(k_{1}, 0\right) \otimes \cdots \otimes L_{\mathfrak{g}_{s}}\left(k_{s}, 0\right) \otimes V_{L},
$$

where the commutant $U^{c}$ of $U$ in $V$ is a vertex operator superalgebra such that $U_{1}^{c}=0$.

\section{References}

[Abe 2005] T. Abe, "Rationality of the vertex operator algebra $V_{L}^{+}$for a positive definite even lattice L”, Math. Z. 249:2 (2005), 455-484. MR 2005k:17033 Zbl 1136.17021

[Borcherds 1986] R. E. Borcherds, "Vertex algebras, Kac-Moody algebras, and the monster", Proc. Nat. Acad. Sci. U.S.A. 83:10 (1986), 3068-3071. MR 87m:17033 Zbl 0613.17012

[Dijkgraaf et al. 1989] R. Dijkgraaf, C. Vafa, E. Verlinde, and H. Verlinde, "The operator algebra of orbifold models", Comm. Math. Phys. 123:3 (1989), 485-526. MR 91c:81132 Zbl 0674.46051

[Dong and Griess 2002] C. Dong and R. L. Griess, Jr., "Automorphism groups and derivation algebras of finitely generated vertex operator algebras", Michigan Math. J. 50:2 (2002), 227-239. MR 2003e:17043 Zbl 1038.17020

[Dong and Lepowsky 1993] C. Dong and J. Lepowsky, Generalized vertex algebras and relative vertex operators, Progress in Mathematics 112, Birkhäuser, Boston, MA, 1993. MR 95b:17032 Zbl 0803.17009

[Dong and Mason 2004] C. Dong and G. Mason, "Rational vertex operator algebras and the effective central charge", Int. Math. Res. Not. 2004:56 (2004), 2989-3008. MR 2005k:17034 Zbl 1106. 17032 
[Dong and Mason 2006] C. Dong and G. Mason, "Integrability of $C_{2}$-cofinite vertex operator algebras”, Int. Math. Res. Not. 2006 (2006), Art. ID 80468. MR 2007c:17036 Zbl 1106.17033

[Dong and Zhang 2008] C. Dong and W. Zhang, "Rational vertex operator algebras are finitely generated", J. Algebra 320:6 (2008), 2610-2614. MR 2009e:17053 Zbl 1233.17023

[Dong and Zhao 2005] C. Dong and Z. Zhao, "Modularity in orbifold theory for vertex operator superalgebras", Comm. Math. Phys. 260:1 (2005), 227-256. MR 2007a:17041 Zbl 1133.17016

[Dong and Zhao 2006] C. Dong and Z. Zhao, "Twisted representations of vertex operator superalgebras”, Commun. Contemp. Math. 8:1 (2006), 101-121. MR 2007b:17038 Zbl 1130.17012

[Dong et al. 1997] C. Dong, H. Li, and G. Mason, "Regularity of rational vertex operator algebras", Adv. Math. 132:1 (1997), 148-166. MR 98m:17037 Zbl 0902.17014

[Dong et al. 1998a] C. Dong, H. Li, and G. Mason, "Twisted representations of vertex operator algebras", Math. Ann. 310:3 (1998), 571-600. MR 99d:17030 Zbl 0890.17029

[Dong et al. 1998b] C. Dong, H. Li, and G. Mason, "Twisted representations of vertex operator algebras and associative algebras", Int. Math. Res. Not. 1998:8 (1998), 389-397. MR 99f:17032 Zbl 0916.17023

[Dong et al. 1998c] C. Dong, H. Li, and G. Mason, "Vertex operator algebras and associative algebras", J. Algebra 206:1 (1998), 67-96. MR 99i:17029 Zbl 0911.17017

[Dong et al. 2000] C. Dong, H. Li, and G. Mason, "Modular-invariance of trace functions in orbifold theory and generalized moonshine", Comm. Math. Phys. 214:1 (2000), 1-56. MR 2001k:17043 Zbl 1061.17025

[Feingold et al. 1991] A. J. Feingold, I. B. Frenkel, and J. F. X. Ries, Spinor construction of vertex operator algebras, triality, and $E_{8}^{(1)}$, Contemporary Mathematics 121, American Mathematical Society, Providence, RI, 1991. MR 92k:17041 Zbl 0743.17029

[Frenkel and Zhu 1992] I. B. Frenkel and Y. Zhu, "Vertex operator algebras associated to representations of affine and Virasoro algebras", Duke Math. J. 66:1 (1992), 123-168. MR 93g:17045 Zbl 0848.17032

[Frenkel et al. 1988] I. Frenkel, J. Lepowsky, and A. Meurman, Vertex operator algebras and the monster, Pure and Applied Mathematics 134, Academic, Boston, MA, 1988. MR 90h:17026 Zbl 0674.17001

[Frenkel et al. 1993] I. B. Frenkel, Y.-Z. Huang, and J. Lepowsky, On axiomatic approaches to vertex operator algebras and modules, Mem. Amer. Math. Soc. 104, American Mathematical Society, Providence, RI, 1993. MR 94a:17007 Zbl 0789.17022

[Gaberdiel and Neitzke 2003] M. R. Gaberdiel and A. Neitzke, "Rationality, quasirationality and finite W-algebras", Comm. Math. Phys. 238:1-2 (2003), 305-331. MR 2004g:81084 Zbl 1042.17025

[Kac 1990] V. G. Kac, Infinite-dimensional Lie algebras, 3rd ed., Cambridge University Press, 1990. MR 92k:17038 Zbl 0716.17022

[Kac and Wang 1994] V. Kac and W. Wang, "Vertex operator superalgebras and their representations", pp. 161-191 in Mathematical aspects of conformal and topological field theories and quantum groups (South Hadley, MA, 1992), edited by P. Sally, Jr. et al., Contemp. Math. 175, American Mathematical Society, Providence, RI, 1994. MR 95k:17040 Zbl 0838.17035

[Karel and Li 1999] M. Karel and H. Li, "Certain generating subspaces for vertex operator algebras", J. Algebra 217:2 (1999), 393-421. MR 2001i:17034 Zbl 0951.17012

[Knopp 1970] M. I. Knopp, Modular functions in analytic number theory, Markham, Chicago, IL, 1970. MR 42 \#198 Zbl 0259.10001 
[Knopp and Mason 2003] M. Knopp and G. Mason, "On vector-valued modular forms and their Fourier coefficients”, Acta Arith. 110:2 (2003), 117-124. MR 2004j:11043 Zbl 1044.11020

[Lepowsky and Li 2004] J. Lepowsky and H. Li, Introduction to vertex operator algebras and their representations, Progress in Mathematics 227, Birkhäuser, Boston, MA, 2004. MR 2004k:17050 Zbl 1055.17001

[Li 1994] H. S. Li, "Symmetric invariant bilinear forms on vertex operator algebras", J. Pure Appl. Algebra 96:3 (1994), 279-297. MR 96e:17063 Zbl 0813.17020

[Li 1996a] H.-S. Li, "Local systems of twisted vertex operators, vertex operator superalgebras and twisted modules", pp. 203-236 in Moonshine, the monster, and related topics (South Hadley, MA, 1994), Contemp. Math. 193, American Mathematical Society, Providence, RI, 1996. MR 1372724 (96m:17050) Zbl 0844.17022

[Li 1996b] H.-S. Li, "Local systems of vertex operators, vertex superalgebras and modules", J. Pure Appl. Algebra 109:2 (1996), 143-195. MR 97d:17016 Zbl 0854.17035

[Mason 2011] G. Mason, "Lattice subalgebras of strongly regular vertex operator algebras", preprint, 2011. arXiv 1110.0544

[Xu 1998] X. Xu, Introduction to vertex operator superalgebras and their modules, Mathematics and its Applications 456, Kluwer, Dordrecht, 1998. MR 2000h:17019 Zbl 0929.17030

[Zhu 1996] Y. Zhu, "Modular invariance of characters of vertex operator algebras", J. Amer. Math. Soc. 9:1 (1996), 237-302. MR 96c:17042 Zbl 0854.17034

Received November 23, 2011. Revised January 25, 2012.

\section{ChONGYING DONG}

DEPARTMENT OF MATHEMATICS

UC SANTA CRUZ

194 BASKIN ENGINEERING

SANTA CRUZ, CALIFORNIA 95064

UNITED STATES

dong@ucsc.edu

\section{JIANZHI HAN}

Wu Wen-Tsun Key Laboratory of Mathematics

USTC

Chinese ACAdemy of Sciences, Hefei 230026

CHINA

and

DEPARTMENT OF MATHEMATICS

SICHUAN UNIVERSITY

CHENGDU, 610064

CHINA

jzzhan@mail.ustc.edu.cn 


\title{
PACIFIC JOURNAL OF MATHEMATICS
}

\author{
http://pacificmath.org \\ Founded in 1951 by \\ E. F. Beckenbach (1906-1982) and F. Wolf (1904-1989)
}

\section{EDITORS}

V. S. Varadarajan (Managing Editor)

Department of Mathematics

University of California

Los Angeles, CA 90095-1555

pacific@math.ucla.edu

Vyjayanthi Chari

Department of Mathematics

University of California

Riverside, CA 92521-0135

chari@math.ucr.edu

\section{Robert Finn}

Department of Mathematics Stanford University

Stanford, CA 94305-2125

finn@math.stanford.edu

Kefeng Liu

Department of Mathematics

University of California

Los Angeles, CA 90095-1555

liu@math.ucla.edu
Darren Long

Department of Mathematics

University of California

Santa Barbara, CA 93106-3080

long@math.ucsb.edu

Jiang-Hua Lu

Department of Mathematics

The University of Hong Kong

Pokfulam Rd., Hong Kong jhlu@maths.hku.hk

Alexander Merkurjev

Department of Mathematics

University of California

Los Angeles, CA 90095-1555

merkurev@math.ucla.edu
Sorin Popa

Department of Mathematics University of California

Los Angeles, CA 90095-1555 popa@math.ucla.edu

Jie Qing

Department of Mathematics

University of California

Santa Cruz, CA 95064

qing@cats.ucsc.edu

Jonathan Rogawski

Department of Mathematics

University of California

Los Angeles, CA 90095-1555

jonr@math.ucla.edu

\section{PRODUCTION}

pacific@math.berkeley.edu

\section{SUPPORTING INSTITUTIONS}

ACADEMIA SINICA, TAIPEI

CALIFORNIA INST. OF TECHNOLOGY INST. DE MATEMÁTICA PURA E APLICADA KEIO UNIVERSITY

MATH. SCIENCES RESEARCH INSTITUTE NEW MEXICO STATE UNIV.

OREGON STATE UNIV.

\author{
STANFORD UNIVERSITY \\ UNIV. OF BRITISH COLUMBIA \\ UNIV. OF CALIFORNIA, BERKELEY \\ UNIV. OF CALIFORNIA, DAVIS \\ UNIV. OF CALIFORNIA, LOS ANGELES \\ UNIV. OF CALIFORNIA, RIVERSIDE \\ UNIV. OF CALIFORNIA, SAN DIEGO \\ UNIV. OF CALIF., SANTA BARBARA
}

\author{
UNIV. OF CALIF., SANTA CRUZ \\ UNIV. OF MONTANA \\ UNIV. OF OREGON \\ UNIV. OF SOUTHERN CALIFORNIA \\ UNIV. OF UTAH \\ UNIV. OF WASHINGTON \\ WASHINGTON STATE UNIVERSITY
}

These supporting institutions contribute to the cost of publication of this Journal, but they are not owners or publishers and have no responsibility for its contents or policies.

See inside back cover or pacificmath.org for submission instructions.

The subscription price for 2012 is US \$420/year for the electronic version, and \$485/year for print and electronic.

Subscriptions, requests for back issues from the last three years and changes of subscribers address should be sent to Pacific Journal of Mathematics, P.O. Box 4163, Berkeley, CA 94704-0163, U.S.A. Prior back issues are obtainable from Periodicals Service Company, 11 Main Street, Germantown, NY 12526-5635. The Pacific Journal of Mathematics is indexed by Mathematical Reviews, Zentralblatt MATH, PASCAL CNRS Index, Referativnyi Zhurnal, Current Mathematical Publications and the Science Citation Index.

The Pacific Journal of Mathematics (ISSN 0030-8730) at the University of California, c/o Department of Mathematics, 969 Evans Hall, Berkeley, CA 94720-3840, is published monthly except July and August. Periodical rate postage paid at Berkeley, CA 94704, and additional mailing offices. POSTMASTER: send address changes to Pacific Journal of Mathematics, P.O. Box 4163, Berkeley, CA 94704-0163.

PJM peer review and production are managed by EditFLOW ${ }^{\mathrm{TM}}$ from Mathematical Sciences Publishers.

PUBLISHED BY PACIFIC JOURNAL OF MATHEMATICS

at the University of California, Berkeley 94720-3840

A NON-PROFIT CORPORATION

Typeset in LATEX

Copyright $(02012$ by Pacific Journal of Mathematics 


\section{PACIFIC JOURNAL OF MATHEMATICS}

Volume $258 \quad$ No. $2 \quad$ August 2012

Uniqueness theorems for $\mathrm{CR}$ and conformal mappings

YounG-JUn CHOI and JAE-CHEON JOO

Some finite properties for vertex operator superalgebras

269

CHONGYING DONG and JIANZHI HAN

On the geometric flows solving Kählerian inverse $\sigma_{k}$ equations

291

HAO FANG and MIJIA LAI

An optimal anisotropic Poincaré inequality for convex domains

GUOFANG WANG and CHAO XIA

Einstein metrics and exotic smooth structures

MASASHI ISHIDA

Noether's problem for $\hat{S}_{4}$ and $\hat{S}_{5}$

MING-CHANG KANG and JiAn ZHOU

Remarks on the behavior of nonparametric capillary surfaces at corners 369

KIRK E. LANCASTER

Generalized normal rulings and invariants of Legendrian solid torus links

MIKHAIL LAVROV and DAN RUTHERFORD

Classification of singular $\mathbb{Q}$-homology planes II: $\mathbb{C}^{1}$ - and $\mathbb{C}^{*}$-rulings.

KAROL PALKA

A dynamical interpretation of the profile curve of CMC twizzler surfaces

OSCAR M. PERDOMO

Classification of Ising vectors in the vertex operator algebra $V_{L}^{+}$

HIROKI SHIMAKURA

Highest-weight vectors for the adjoint action of $\mathrm{GL}_{n}$ on polynomials 